\title{
Magnitude and causes of socioeconomic differentials in mortality: further evidence from the Whitehall Study
}

\author{
George Davey Smith, Martin J Shipley, Geoffrey Rose
}

\begin{abstract}
Study objective-The aim was to explore the magnitude and causes of the differences in mortality rates according to socioeconomic position in a cohort of civil servants.
\end{abstract}

Design-This was a prospective observational study of civil servants followed up for 10 years after baseline data collection.

Setting-Civil service offices in London. Participants-11 678 male civil servants were studied, aged $40-64$ at baseline screening between 1967 and 1969. Two indices of socioeconomic position were available on these participants-employment grade (categorised into four levels), and ownership of a car.

Measurements and main results-Main outcome measures were all cause and cause specific mortality, with cause of death taken from death certificates coded according to the eighth revision of the ICD. Employment grade and car ownership were independently related to total mortality and to mortality from the major cause groups. Combining the indices further improved definition of mortality risk and the age adjusted relative rate between the highest grade car owners and the lowest grade nonowners of 4.3 is considerably larger than the social class differentials seen in the British population. Factors potentially involved in the production of these mortality differentials were examined. Smoking, plasma cholesterol concentration, blood pressure, and glucose intolerance did not appear to account for them. The pattern of differentials was the same in the group who reported no ill health at baseline as it was in the whole sample, which suggests that health selection associated with frank illness was not a major determinant. The contribution of height, a marker for environmental factors acting in early life, was also investigated. Whereas adjustment for employment grade and car ownership attenuated the association between short stature and mortality, height differences within employment grade and car ownership groups explained little of the differential mortality.

Conclusion-The use of social class as an index of socioeconomic position leads to underestimation of the association between social factors and mortality, which may be reflected in public health initiatives and priorities. Known risk factors could not be shown to account for the differentials in mortality, although the degree to which this can be explored with single measurements is limited.

Since the 1911 census the classification of occupations into the Registrar General's social classes (RGSC) has been the basis for the examination of socioeconomic differentials in mortality rates. ${ }^{1}$ As the inventor of this system made clear, it was intended to categorise people according to their degree of material well being among other factors. ${ }^{23}$ Since then information generated from the analysis of mortality according to RGSC has been used to support the notion that lower living standards are associated with higher death rates. ${ }^{3-5}$

The appropriateness of the RGSC for examining socioeconomic differences in mortality has been questioned and defended from several standpoints. ${ }^{6-9}$ This categorisation is imprecise in regard to income-within class variation in income is greater than that between classes. ${ }^{10}$ Other factors within the social environment will also be distributed more unevenly than the Registrar General's social class categorisation would suggest. Thus estimates from social class analysis will attenuate the underlying associations between material and social well being and mortality. ${ }^{11}$ The OPCS Longitudinal Study has explored this by combining other indicators of socioeconomic position-housing tenure and occupancy, possession of household amenities, car ownership, and level of education-with the Registrar General's social classes in the examination of mortality and cancer registration rates. ${ }^{12-14}$ In general, being an owner-occupier, possessing household amenities and a car, having had higher education and being in a higher social class group were related to lower mortality and cancer registration rates. When these classifications were combined, independent effects were seen. This suggests that the effects of socioeconomic environment upon health are not adequately characterised by the use of social class categories alone. Better discrimination is achieved by combining indicators of socioeconomic position.

Complementary findings have come from follow up of a cohort study of civil servants, the Whitehall Study, in which employment grade has served as the marker of socioeconomic position. ${ }^{15-17}$ The differences in 10 year mortality rates between grades were considerably greater than the Decennial Supplements suggested. ${ }^{10}$

It is of interest to see if further refinement of categorisation in the Whitehall Study leads to 
increases in differentials. The availability of data regarding car ownership allows this to be examined. Having access to a car was the strongest independent predictor of mortality among men who could be assigned to a social class in the Longitudinal Study. ${ }^{18}$ Thus the combination of employment grade and car ownership provides a test of the hypothesis that more precise classification of socioeconomic position increases the mortality differentials observed.

The present study examines the independent and combined effects of employment grade and car ownership on mortality. The 10 year follow up data are examined, as mortality over this period corresponds best with the Decennial Supplements, which only cover men aged 64 years and under. Analyses are repeated after excluding men sick at the time of screening, to allow the effects of selection to be explored. The degree to which differences in smoking, blood pressure, plasma cholesterol concentration, and glucose tolerance can explain mortality differentials is also examined.

It has been postulated that factors acting during early life are related to the risk of cardiovascular disease in adulthood. ${ }^{19}$ Height is influenced both by genetic factors and by childhood environment. ${ }^{2021}$ Short stature is associated with higher mortality risk in the Whitehall study, ${ }^{17}$ a finding in line with other investigations which have related markers of early environmental conditions to mortality. 2223 The possible role of conditions in childhood, as indexed by height, in the generation of socioeconomic position differentials in mortality in adulthood is investigated in the present cohort.

\section{Methods}

In the Whitehall study 18403 men aged 40-64 were examined between 1967 and 1969. ${ }^{24}$ Measurements included height, weight, blood pressure, a six lead electrocardiogram, plasma cholesterol concentration, and a glucose tolerance test. A questionnaire was completed regarding age, civil service employment grade, smoking habits, and health status.

The electrocardiogram was coded according to the Minnesota system ${ }^{25}$ and was regarded as positive for ischaemia if $\mathrm{Q} / \mathrm{QS}$ items (codes $1 \cdot 1-3$ ), ST/T items (codes $4 \cdot 1-4$ or $5 \cdot 1-3$ ), or left bundle branch block (code 7.1) were present. Subjects with blood glucose two hours after a post-fasting $50 \mathrm{~g}$ glucose load $\geqslant 11 \cdot 1 \mathrm{mmol} /$ litre ( $\geqslant 200 \mathrm{mg} / 100 \mathrm{ml}$ ) or with previously diagnosed diabetes constituted the diabetic group; nondiabetic subjects with glucose concentrations above the 95 th centile point $(5.4-11.0 \mathrm{mmol} /$ litre; $96-199 \mathrm{mg} / 100 \mathrm{ml}$ ) formed the group with impaired glucose tolerance, and other subjects were designated as being normoglycaemic.

The questionnaire used in the study was modified at various times, the details of car ownership and employment grade having been obtained from the 11678 subjects who were seen in the middle period of the survey. This group forms the basis for the analysis. Employment grade was categorised as administrative, professional or executive, clerical, and "other grades" (men in messenger and other unskilled manual jobs). Smoking has been categorised according to cigarette use as "current smoker", "ex-smoker" and "never smoker". The 409 men who smoked pipes or cigars only have been excluded from the analyses that involve smoking status.

Data were missing for car ownership (5), blood pressure (3), cholesterol (556), glucose tolerance (90), body mass index (2), and smoking status (3). Subjects were only excluded from the analyses for which their specific data were missing.

Records from over $99 \%$ of subjects were traced and flagged at the National Health Service Central Registry. Death certificates coded according to the eighth revision of the International Classification of Diseases (ICD) have been obtained, and this almost complete mortality follow up provides the basis for this analysis. Mortality has been classified as being due to cardiovascular disease (CVD; ICD codes 390-458), neoplasms (ICD codes 140-239), or other causes. For seven deaths the cause was unknown. These have been included in all cause mortality, but have been excluded from analyses of cause specific mortality. Mortality causes have also been classified according to whether smoking is considered to play a role in their aetiology. ${ }^{26-29}$ The causes deemed to be smoking related, with their ICD codes, are: malignant neoplasms of the lip (ICD 140), tongue (141), mouth and pharynx (143-149), oesophagus (150), pancreas (157), respiratory system (160-163), and urinary system (188-189); hypertensive disease (400-404); ischaemic heart disease (410-414); pulmonary heart disease (426); cerebrovascular disease (430438); diseases of the arteries, arterioles and capillaries (440-448); pneumonia (480-486); bronchitis and emphysema (491-492); and peptic ulcer (531-534).

Mortality rates have been calculated using person years at risk. These rates and also all means and proportions have been standardised for age at entry by the direct method, using the study population as the standard. Adjustment for other major risk factors and calculation of confidence intervals for the relative risks was done using Cox's proportional hazards regression model..$^{30}$ The associations between grade and risk factors were examined by fitting a trend term for grade in a regression model of the variable of interest, while adjusting for age and car ownership. These were computed using multiple regression for the continuous variables and log linear models for the discrete factors.

\section{Results}

Seventy two percent of the study population owned a car, percentages falling from $91 \%(600 / 658)$ in administrators, through $82 \%(6877 / 8387)$ in professionals/executives and $39 \%(665 / 1712)$ in clerical staff, to $34 \%$ (307/916) among other grades. The small number of administrators without a car produces only imprecise estimates of rates.

Table I presents age standardised 10 year mortality rates according to employment grade and car ownership. Each is independently and consistently related to all cause mortality rates. The relationship is also seen for the major groups of causes and for causes unrelated to smoking, 
though there is some loss of consistency, perhaps due to the small number of deaths in some categories.

After adjusting for age the relative mortality rate associated with not owning a car was 1.49 (95\% confidence interval $1 \cdot 4-1 \cdot 7)$; adjusting for employment grade reduced this to $1.28(1 \cdot 1-1 \cdot 5)$. Compared to the professional or executives, the age adjusted relative mortality rates for the administrators, clerical and other grades were $0.51(0.3-0.8), 1.43(1.2-1.7)$, and 1.64 (1.4-2.0) respectively. After adjusting for car ownership these became $0.52(0.3-0.8), 1.29(1.1-1.5)$ and $1.46(1 \cdot 2-1 \cdot 8)$. Thus, while car ownership and grade are associated, much of their relationship with mortality is independent of one another.

Employment grade and car ownership were related to indicators of health status and to risk factors for cardiovascular disease. Age adjusted values according to employment grade and car ownership are presented in tables II and III. Independent associations were examined, and

Table I Age adjusted 10 year mortality rates with their standard errors and number of deaths, by employment grade and car ownership status

\begin{tabular}{|c|c|c|c|c|c|c|c|}
\hline & \multirow[b]{3}{*}{$\begin{array}{l}\text { Employment } \\
\text { grade }\end{array}$} & \multicolumn{6}{|c|}{ Car owner } \\
\hline & & \multicolumn{3}{|l|}{ Yes } & \multicolumn{3}{|l|}{ No } \\
\hline & & Rate $^{a}$ & $(S E)^{b}$ & $\begin{array}{l}\text { No of } \\
\text { deaths }\end{array}$ & Rate & $(S E)$ & $\begin{array}{l}\text { No of } \\
\text { deaths }\end{array}$ \\
\hline \multirow[t]{2}{*}{ All causes } & $\begin{array}{l}\text { Administrative } \\
\text { Professional or executive } \\
\text { Clerical } \\
\text { Other }\end{array}$ & $\begin{array}{r}4 \cdot 4 \\
8 \cdot 5 \\
10 \cdot 8 \\
11 \cdot 9\end{array}$ & $\begin{array}{l}(1 \cdot 0) \\
(0 \cdot 4) \\
(1 \cdot 2) \\
(2 \cdot 1)\end{array}$ & $\begin{array}{r}21 \\
496 \\
79 \\
42\end{array}$ & $\begin{array}{r}4 \cdot 9 \\
10 \cdot 7 \\
14 \cdot 2 \\
18 \cdot 8\end{array}$ & $\begin{array}{l}(2 \cdot 9) \\
(0.9) \\
(1 \cdot 2) \\
(2 \cdot 0)\end{array}$ & $\begin{array}{r}3 \\
147 \\
165 \\
133\end{array}$ \\
\hline & Total $^{c}$ & $9 \cdot 2$ & $(0 \cdot 4)$ & 638 & 11.9 & $(0.6)$ & 448 \\
\hline \multirow[t]{2}{*}{$\begin{array}{l}\text { Cardiovascular } \\
\text { disease }\end{array}$} & $\begin{array}{l}\text { Administrative } \\
\text { Professional or executive } \\
\text { Clerical } \\
\text { Other }\end{array}$ & $\begin{array}{l}3 \cdot 3 \\
4 \cdot 9 \\
5 \cdot 8 \\
6 \cdot 7\end{array}$ & $\begin{array}{l}(0.9) \\
(0.3) \\
(0.9) \\
(1 \cdot 6)\end{array}$ & $\begin{array}{r}14 \\
281 \\
42 \\
23\end{array}$ & $\begin{array}{l}1 \cdot 5 \\
5 \cdot 7 \\
7 \cdot 4 \\
9 \cdot 8\end{array}$ & $\begin{array}{l}(1 \cdot 5) \\
(0.7) \\
(0.9) \\
(1 \cdot 4)\end{array}$ & $\begin{array}{r}1 \\
79 \\
85 \\
73\end{array}$ \\
\hline & Total $^{c}$ & $5 \cdot 2$ & $(0 \cdot 3)$ & 360 & $6 \cdot 3$ & $(0.5)$ & 238 \\
\hline \multirow[t]{2}{*}{ Cancer } & $\begin{array}{l}\text { Administrative } \\
\text { Professional or executive } \\
\text { Clerical } \\
\text { Other }\end{array}$ & $\begin{array}{l}1 \cdot 1 \\
2 \cdot 6 \\
3 \cdot 2 \\
3 \cdot 7\end{array}$ & $\begin{array}{l}(0 \cdot 4) \\
(0 \cdot 2) \\
(0 \cdot 7) \\
(1 \cdot 1)\end{array}$ & $\begin{array}{r}7 \\
156 \\
24 \\
14\end{array}$ & $\begin{array}{l}3 \cdot 5 \\
3 \cdot 3 \\
4 \cdot 6 \\
4 \cdot 6\end{array}$ & $\begin{array}{l}(2 \cdot 4) \\
(0.5) \\
(0.7) \\
(0.9)\end{array}$ & $\begin{array}{r}2 \\
45 \\
54 \\
33\end{array}$ \\
\hline & Total $^{c}$ & $2 \cdot 9$ & $(0 \cdot 2)$ & 201 & 3.7 & $(0 \cdot 4)$ & 134 \\
\hline \multirow[t]{2}{*}{ Other causes } & $\begin{array}{l}\text { Administrative } \\
\text { Professional or executive } \\
\text { Clerical } \\
\text { Other }\end{array}$ & $\begin{array}{l}0.0 \\
1 \cdot 0 \\
1 \cdot 7 \\
0.9\end{array}$ & $\begin{array}{l}(-) \\
(0 \cdot 1) \\
(0.5) \\
(0 \cdot 4)\end{array}$ & $\begin{array}{r}0 \\
57 \\
13 \\
4\end{array}$ & $\begin{array}{l}0 \cdot 0 \\
1 \cdot 5 \\
2 \cdot 2 \\
4 \cdot 2\end{array}$ & $\begin{array}{l}(-) \\
(0.3) \\
(0.5) \\
(1.0)\end{array}$ & $\begin{array}{r}0 \\
21 \\
25 \\
26\end{array}$ \\
\hline & Total $^{c}$ & $1 \cdot 1$ & $(0 \cdot 1)$ & 74 & 1.8 & $(0 \cdot 2)$ & 72 \\
\hline \multirow[t]{2}{*}{$\begin{array}{l}\text { Non-smoking } \\
\text { related causes }\end{array}$} & $\begin{array}{l}\text { Administrative } \\
\text { Professional or executive } \\
\text { Clerical } \\
\text { Other }\end{array}$ & $\begin{array}{l}0.7 \\
2 \cdot 2 \\
2 \cdot 9 \\
1.9\end{array}$ & $\begin{array}{l}(0 \cdot 3) \\
(0 \cdot 2) \\
(0 \cdot 6) \\
(0 \cdot 7)\end{array}$ & $\begin{array}{r}5 \\
134 \\
21 \\
9\end{array}$ & $\begin{array}{l}1 \cdot 7 \\
2 \cdot 7 \\
3 \cdot 9 \\
3 \cdot 7\end{array}$ & $\begin{array}{l}(1 \cdot 7) \\
(0.4) \\
(0.6) \\
(0.9)\end{array}$ & $\begin{array}{r}1 \\
39 \\
43 \\
25\end{array}$ \\
\hline & Total $^{c}$ & $2 \cdot 2$ & $(0 \cdot 2)$ & 169 & 3.4 & $(0 \cdot 3)$ & 108 \\
\hline
\end{tabular}

a Rates per 1000 person years

cAdjusted for employment grade and age after adjusting for car ownership, lower work grade was significantly associated $(p<0.05)$ with higher systolic blood pressure and body mass index, lower plasma cholesterol concentration and shorter height, and higher prevalence of smoking, glucose intolerance or being diabetic, and having disease at baseline (abnormal electrocardiogram, angina, intermittent claudication, dyspnoea, or being under medical care for hypertension or heart disease). Independently of employment grade, non-ownership of a car was significantly associated with lower height and body mass index, higher systolic blood pressure, and higher prevalence of smoking and disease at baseline.

Smoking, pre-existing disease, and other risk factors might therefore account for the socioeconomic differences in mortality. Table I shows that these differences are seen for mortality from causes not related to smoking. Within subjects who had never smoked, the same consistent pattern of all cause mortality rates by grade and car ownership as exists in the whole cohort is seen, although this is based on only 114 deaths. Furthermore, excluding the $22 \%$ of subjects with disease at entry has little effect on the pattern of mortality differentials.

Relative rates were calculated with the largest group-professional or executive grade car owners-as the baseline category. Relative rates for cardiovascular disease and all causes were adjusted for age, smoking (category and number of cigarettes smoked), systolic blood pressure, plasma cholesterol concentration, and glucose intolerance. Relative rates for neoplasms and other causes were adjusted for age and smoking. The independent associations of grade and car ownership with mortality rates are not greatly affected by these adjustments (table IV).

Height, which is inversely associated to all cause mortality, coronary heart disease (CHD) and non-CHD mortality in this cohort, ${ }^{17}$ was related to employment grade and car ownership. The relative mortality rates for the lowest two tertiles of height versus the top tertile are shown in table V. Lower height is significantly $(p<0.05$ related to increased rates of all cause, cardiovascular, and non-cardiovascular nonneoplastic mortality, but not to mortality from neoplasms. Adjusting for grade and car ownership reduced the strength of, but did not abolish, the associations between height and mortality. Adjustment for grade and car ownership together resulted in greater reduction in the relative rates than did adjustment for just one of these factors.
Table II Means and standard errors for major risk factors (ageadjusted) by employment grade and car ownership

\begin{tabular}{|c|c|c|c|c|c|c|c|c|c|}
\hline \multirow[b]{3}{*}{$\begin{array}{l}\text { Systolic blood } \\
\text { pressure (mm Hg) }\end{array}$} & \multirow[b]{3}{*}{$\begin{array}{l}\text { Car owner } \\
\text { No car }\end{array}$} & \multicolumn{8}{|c|}{ Employment grade } \\
\hline & & \multicolumn{2}{|c|}{$\begin{array}{l}\text { Administrative } \\
\text { Mean (SE) }\end{array}$} & \multicolumn{2}{|c|}{$\begin{array}{l}\text { Professional } \\
\text { or executive } \\
\text { Mean }(S E)\end{array}$} & \multicolumn{2}{|c|}{$\begin{array}{l}\text { Clerical } \\
\text { Mean (SE) }\end{array}$} & \multicolumn{2}{|c|}{$\begin{array}{l}\text { Other } \\
\text { Mean (SE) }\end{array}$} \\
\hline & & $\begin{array}{l}133.3 \\
133.8\end{array}$ & $\begin{array}{l}(0.94) \\
(2.43)\end{array}$ & $\begin{array}{l}135 \cdot 2 \\
136.8\end{array}$ & $\begin{array}{l}(0.25) \\
(0.52)\end{array}$ & $\begin{array}{l}136.0 \\
135.9\end{array}$ & $\begin{array}{l}(0.83) \\
(0.68)\end{array}$ & $\begin{array}{l}137 \cdot 2 \\
136 \cdot 2\end{array}$ & $\begin{array}{l}(1 \cdot 21) \\
(1 \cdot 12)\end{array}$ \\
\hline $\begin{array}{l}\text { Diastolic blood } \\
\text { pressure (mm Hg) }\end{array}$ & $\begin{array}{l}\text { Car owner } \\
\text { No car }\end{array}$ & $\begin{array}{l}84 \cdot 0 \\
83 \cdot 8\end{array}$ & $\begin{array}{l}(0.61) \\
(1.83)\end{array}$ & $\begin{array}{l}84 \cdot 2 \\
84.8\end{array}$ & $\begin{array}{l}(0 \cdot 17) \\
(0 \cdot 35)\end{array}$ & $\begin{array}{l}84 \cdot 2 \\
84 \cdot 6\end{array}$ & $\begin{array}{l}(0.58) \\
(0.4)\end{array}$ & $\begin{array}{l}86 \cdot 5 \\
84.5\end{array}$ & $\begin{array}{l}(0.92) \\
(0.72)\end{array}$ \\
\hline $\begin{array}{l}\text { Plasma cholesterol } \\
\text { concentration } \\
\text { (mmol/litre) }\end{array}$ & $\begin{array}{l}\text { Car owner } \\
\text { No car }\end{array}$ & $\begin{array}{l}5 \cdot 32 \\
5 \cdot 37\end{array}$ & $\begin{array}{l}(0 \cdot 06) \\
(0 \cdot 17)\end{array}$ & $\begin{array}{l}5 \cdot 22 \\
5.23\end{array}$ & $\begin{array}{l}(0.02) \\
(0.03)\end{array}$ & $\begin{array}{l}5 \cdot 23 \\
5 \cdot 27\end{array}$ & $\begin{array}{l}(0.05) \\
(0.05)\end{array}$ & $\begin{array}{l}5.12 \\
5.05\end{array}$ & $\begin{array}{l}(0.08) \\
(0.07)\end{array}$ \\
\hline $\begin{array}{l}\text { Body mass } \\
\text { index }\left(\mathbf{k g} / \mathbf{m}^{2}\right)\end{array}$ & $\begin{array}{l}\text { Car owner } \\
\text { No car }\end{array}$ & $\begin{array}{l}24.5 \\
24.8\end{array}$ & $\begin{array}{l}(0.12) \\
(0.33)\end{array}$ & $\begin{array}{l}24 \cdot 8 \\
24 \cdot 6\end{array}$ & $\begin{array}{l}(0.03) \\
(0.08)\end{array}$ & $\begin{array}{l}24 \cdot 8 \\
24.5\end{array}$ & $\begin{array}{l}(0.12) \\
(0.11)\end{array}$ & $\begin{array}{l}25 \cdot 5 \\
24 \cdot 8\end{array}$ & $\begin{array}{l}(0.21) \\
(0.17)\end{array}$ \\
\hline Height (m) & $\begin{array}{l}\text { Car owner } \\
\text { No car }\end{array}$ & $\begin{array}{l}1.78 \\
1.76 \\
\end{array}$ & $\begin{array}{l}(0.003) \\
(0.008)\end{array}$ & $\begin{array}{l}1.764 \\
1.755\end{array}$ & $\begin{array}{l}(0.001) \\
(0.002)\end{array}$ & $\begin{array}{l}1.74 \\
1.73\end{array}$ & $\begin{array}{l}4(0.003) \\
7(0.002)\end{array}$ & $\begin{array}{l}1.754 \\
1.722\end{array}$ & $\begin{array}{l}4(0.005) \\
2(0.004)\end{array}$ \\
\hline
\end{tabular}


Table III Prevalence (age adjusted) of risk factors by employment grade and car ownership

\begin{tabular}{|c|c|c|c|c|c|c|c|c|c|}
\hline & & \multicolumn{8}{|c|}{ Employment grade } \\
\hline & & \multicolumn{2}{|c|}{$\begin{array}{l}\text { Administrative } \\
\% \quad(n)\end{array}$} & \multicolumn{2}{|c|}{$\begin{array}{l}\text { Professional } \\
\text { or executive } \\
\% \quad(n)\end{array}$} & \multicolumn{2}{|l|}{$\begin{array}{l}\text { Clerical } \\
\%\end{array}$} & $\begin{array}{l}\text { Other } \\
\%\end{array}$ & \multirow{2}{*}{$\begin{array}{c}(n) \\
\left.\begin{array}{c}(41) \\
(94) \\
(161)\end{array}\right)\end{array}$} \\
\hline $\begin{array}{l}\text { Car } \\
\text { owner }\end{array}$ & $\begin{array}{l}\text { Non-smokers } \\
\text { Ex-smokers } \\
\text { Current smokers }\end{array}$ & $\begin{array}{l}27 \\
43 \\
30\end{array}$ & $\begin{array}{l}(166) \\
(221) \\
(162)\end{array}$ & $\begin{array}{l}21 \\
43 \\
37\end{array}$ & $\begin{array}{l}(1421) \\
(2788) \\
(2417)\end{array}$ & $\begin{array}{l}15 \\
34 \\
51\end{array}$ & $\begin{array}{l}(94) \\
(230) \\
(327)\end{array}$ & $\begin{array}{l}15 \\
30 \\
55\end{array}$ & \\
\hline No car & $\begin{array}{l}\text { Non-smokers } \\
\text { Ex-smokers } \\
\text { Current smokers }\end{array}$ & $\begin{array}{l}23 \\
30 \\
48\end{array}$ & $\begin{array}{l}(13) \\
(17) \\
(26)\end{array}$ & $\begin{array}{l}20 \\
34 \\
46\end{array}$ & $\begin{array}{l}(299) \\
(488) \\
(671)\end{array}$ & $\begin{array}{l}17 \\
29 \\
54\end{array}$ & $\begin{array}{l}(162) \\
(308) \\
(559)\end{array}$ & $\begin{array}{l}13 \\
20 \\
67\end{array}$ & $\begin{array}{l}(69) \\
(131) \\
(396)\end{array}$ \\
\hline $\begin{array}{l}\text { Car } \\
\text { owner }\end{array}$ & $\begin{array}{l}\text { No disease at entry } \\
\text { Disease at entry }\end{array}$ & $\begin{array}{l}83 \\
17\end{array}$ & $\begin{array}{r}(504) \\
(96)\end{array}$ & $\begin{array}{l}79 \\
21\end{array}$ & $\begin{array}{l}(5517) \\
(1360)\end{array}$ & $\begin{array}{l}79 \\
21\end{array}$ & $\begin{array}{l}(520) \\
(145)\end{array}$ & $\begin{array}{l}81 \\
19\end{array}$ & $\begin{array}{r}(235) \\
(72)\end{array}$ \\
\hline No car & $\begin{array}{l}\text { No disease at entry } \\
\text { Disease at entry }\end{array}$ & $\begin{array}{l}81 \\
19\end{array}$ & $\begin{array}{l}(47) \\
(11)\end{array}$ & $\begin{array}{l}77 \\
23\end{array}$ & $\begin{array}{r}(1175) \\
(335)\end{array}$ & $\begin{array}{l}74 \\
26\end{array}$ & $\begin{array}{l}(762) \\
(285)\end{array}$ & $\begin{array}{l}70 \\
30\end{array}$ & $\begin{array}{l}(389) \\
(220)\end{array}$ \\
\hline \multirow{2}{*}{$\begin{array}{l}\text { Car } \\
\text { owner }\end{array}$} & \multirow{2}{*}{$\begin{array}{l}\text { Normoglycaemic } \\
\text { Glucose intolerant } \\
\text { or diabetic }\end{array}$} & 94 & $(565)$ & 93 & (6407) & 91 & $(601)$ & 88 & (263) \\
\hline & & 5 & (32) & 7 & $(422)$ & 9 & (58) & 12 & (42) \\
\hline \multirow[t]{2}{*}{ No car } & \multirow{2}{*}{$\begin{array}{l}\text { Normoglycaemic } \\
\text { Glucose intolerant } \\
\text { or diabetic }\end{array}$} & 96 & (56) & 92 & $(1385)$ & 91 & (933) & 91 & (535) \\
\hline & & 4 & (2) & 8 & (140) & 9 & (84) & 9 & (65) \\
\hline
\end{tabular}

Table IV Adjusted relative rates ${ }^{a}$ for 10 year mortality for major causes of death by employment grade and car ownership status (95\% confidence intervals in parentheses)

\begin{tabular}{|c|c|c|c|c|c|}
\hline & & \multicolumn{4}{|c|}{ Employment grade } \\
\hline & & Administrative & $\begin{array}{l}\text { Professional } \\
\text { or executive }\end{array}$ & Clerical & Other \\
\hline All causes & $\begin{array}{l}\text { Car owner } \\
\text { No car }\end{array}$ & $\begin{array}{l}0.60 \\
(0.4-0.9) \\
0 \cdot 71 \\
(0 \cdot 2-2 \cdot 2)\end{array}$ & 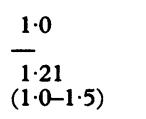 & $\begin{array}{l}1.26 \\
(1.0-1.6) \\
1.45 \\
(1.2-1 \cdot 7)\end{array}$ & $\begin{array}{l}1 \cdot 19 \\
(0 \cdot 9-1 \cdot 6) \\
1 \cdot 60 \\
(1 \cdot 3-2 \cdot 0)\end{array}$ \\
\hline Cardiovascular disease & $\begin{array}{l}\text { Car owner } \\
\text { No car }\end{array}$ & $\begin{array}{l}0.71 \\
(0 \cdot 4-1 \cdot 2) \\
0.40 \\
(0 \cdot 1-2 \cdot 9)\end{array}$ & $\begin{array}{l}1.0 \\
1 \cdot 12 \\
(0.9-1 \cdot 5)\end{array}$ & $\begin{array}{l}1 \cdot 16 \\
(0.8-1 \cdot 6) \\
1 \cdot 27 \\
(1 \cdot 0-1 \cdot 6)\end{array}$ & $\begin{array}{l}1 \cdot 13 \\
(0 \cdot 7-1 \cdot 7) \\
1 \cdot 53 \\
(1 \cdot 2-2 \cdot 0)\end{array}$ \\
\hline Cancer & $\begin{array}{l}\text { Car owner } \\
\text { No car }\end{array}$ & $\begin{array}{l}0.59 \\
(0.3-1 \cdot 3) \\
1 \cdot 37 \\
(0 \cdot 3-5 \cdot 5)\end{array}$ & $\begin{array}{l}\frac{1 \cdot 0}{1 \cdot 16} \\
(0 \cdot 8-1 \cdot 6)\end{array}$ & $\begin{array}{l}1 \cdot 21 \\
(0 \cdot 8-1 \cdot 9) \\
1 \cdot 60 \\
(1 \cdot 2-2 \cdot 2)\end{array}$ & $\begin{array}{l}1 \cdot 33 \\
(0 \cdot 8-2 \cdot 3) \\
1 \cdot 40 \\
(0 \cdot 9-2 \cdot 1)\end{array}$ \\
\hline "Other" causes & $\begin{array}{l}\text { Car owner } \\
\text { No car }\end{array}$ & $\left(\begin{array}{l}0.0 \\
(0.0-)\end{array}\right)$ & $\begin{array}{l}\frac{1 \cdot 0}{1 \cdot 48} \\
(0.9-2 \cdot 4)\end{array}$ & $\begin{array}{l}1 \cdot 80 \\
(1 \cdot 0-3 \cdot 3) \\
2 \cdot 00 \\
(1 \cdot 2-3 \cdot 2)\end{array}$ & $\begin{array}{l}1 \cdot 02 \\
(0 \cdot 4-2 \cdot 8) \\
2 \cdot 84 \\
(1 \cdot 7-4 \cdot 7)\end{array}$ \\
\hline
\end{tabular}

${ }^{a}$ For all causes and cardiovascular disease relative rates are adjusted for age, systolic blood pressure, cholesterol concentration, smoking status, and glucose intolerance. For cancer and "other" causes relative rates are adjusted for age and smoking status.

Table $V$ Relative rates for the lowest two tertiles of height versus the top tertile, with various adjustments

\begin{tabular}{|c|c|c|c|}
\hline & \multicolumn{3}{|c|}{ Adjusted for } \\
\hline & Age & $\begin{array}{l}\text { Age, grade, } \\
\text { and car } \\
\text { ownership }\end{array}$ & $\begin{array}{l}\text { Age, grade, } \\
\text { car ownership, } \\
\text { risk factors }{ }^{a}\end{array}$ \\
\hline $\begin{array}{l}\text { All causes } \\
\text { Height }<173 \mathrm{~cm} \\
173 \mathrm{~cm} \leqslant \mathrm{Ht} \leqslant 178 \mathrm{~cm}\end{array}$ & $\begin{array}{l}1.41 \\
1.14\end{array}$ & $\begin{array}{l}1.28 \\
1 \cdot 11\end{array}$ & $\begin{array}{l}1.26 \\
1.11\end{array}$ \\
\hline $\begin{array}{l}\text { Cardiovascular } \\
\text { Height }<173 \mathrm{~cm} \\
173 \mathrm{~cm} \leqslant \mathrm{Ht} \leqslant 178 \mathrm{~cm}\end{array}$ & $\begin{array}{l}1.57 \\
1.20\end{array}$ & $\begin{array}{l}1.44 \\
1 \cdot 18\end{array}$ & $\begin{array}{l}1.39 \\
1.18\end{array}$ \\
\hline $\begin{array}{l}\text { Neoplasms } \\
\text { Height }<173 \mathrm{~cm} \\
173 \mathrm{~cm} \leqslant \mathrm{Ht} \leqslant 178 \mathrm{~cm}\end{array}$ & $\begin{array}{l}1.01 \\
0.96\end{array}$ & $\begin{array}{l}0.92 \\
0.93\end{array}$ & $\begin{array}{l}0.92 \\
0.93\end{array}$ \\
\hline $\begin{array}{l}\text { Non-cardiovascular, non-neoplastic } \\
\text { Height }<173 \mathrm{~cm} \\
173 \mathrm{~cm} \leqslant \mathrm{Ht} \leqslant 178 \mathrm{~cm}\end{array}$ & $\begin{array}{l}2.36 \\
1.66\end{array}$ & $\begin{array}{l}1.91 \\
1.55\end{array}$ & $\begin{array}{l}1.89 \\
1.56\end{array}$ \\
\hline
\end{tabular}

a Risk factors are (1) for all causes and cardiovascular disease: systolic blood pressure, cholesterol concentration, smoking status and number of cigarettes per day, glucose intolerance; (2) for neoplasms and other causes: smoking status and number of cigarettes per day.

Additional adjustment for risk factors had little effect on the size of the relative rates. Conversely adjustment for height left the relative mortality rates by employment grade and car ownership status virtually unchanged.

\section{Discussion}

Mortality differentials according to civil service employment grade are enhanced by the combination of this measure with details of car ownership. Over the follow up period, at the end of which the cohort was aged 50-74, the mortality rate ratio for "other" grades without a car compared to administrators with a car was $4 \cdot 3$. For clerical staff without a car the corresponding ratio was 3.2. Clerical workers and administrators both fall into non-manual social class groups. In the 1971 Decennial Supplement ${ }^{10}$ the mortality rate ratios for group III non-manual compared to group I were 1.4 in the 5 year age band 40-44, remaining approximately at this level in the bands $45-49$ and 50-54, falling to 1.2 in the bands 55-59 and 60-64 (the oldest available). Clearly categorisation by employment grade and car ownership in these civil servants produces groups with greater differentials in mortality than does the Registrar General's social class system.

In addition to having been used in the Longitudinal Study and the present study, car ownership has served as an indicator of material well being in a complementary series of investigations. In a study of regional mortality rate variation in the north of England, social class composition of the areas accounted for only a part of the variance in rates. ${ }^{31} \mathrm{~A}$ composite indicator of deprivation, which combined car ownership, unemployment rates, house ownership, and overcrowding, accounted for a greater portion of the variance. Car ownership and unemployment were the most important of the four measures of deprivation in this respect. Similarly car ownership, together with other indices of deprivation, can statistically account for the mortality differences between Scotland and England/Wales, ${ }^{32}$ which are partially independent of social class. Such studies have led to the proposal that composite measures of deprivation may better reveal the relationship between socioeconomic position and health than does analysis by social class alone. ${ }^{33}$

In these studies car ownership has been taken to be a proxy measure of income. Among these civil servants it is probable that within any employment grade the car owners earn more, in relation to their needs, than do those without cars. Needs will vary with factors such as family size and number of dependents. Car ownership may index both absolute and relative income level, and thus usefully complement grade (and social class) in the process of socioeconomic stratification. It is not possible to separate out those who choose not to have a car despite adequate resources from those who could not afford to run a car. Clearly the consequences of not owning a car may be very different in these two groups.

Explanations which have been advanced for the social class inequalities in mortality are that they are artefactual or that they are due to health selection, health related behaviours, or material factors. ${ }^{34}$ The major artefact explanation suggests that differential recording of social class at census and at death causes numerator/denominator bias which leads to apparent differentials in the Decennial Supplements. Cohort studies such as the present one and the Longitudinal Study are not subject to this bias. 
The selection hypothesis postulates that when people become unhealthy they tend to move into lower social class groups. ${ }^{35} 36$ Data on changes in civil service employment grade or car ownership were not available in the present study. Excluding subjects with indentifiable disease at the start of the study did not greatly affect the mortality differentials, so grade mobility or selective recruitment of such people is unlikely to be an adequate explanation. Furthermore such exclusions overcompensate for possible selection, since differential prevalence rates of disease in these middle aged men will reflect socioeconomic factors over their life courses. Other studies, including the Longitudinal Study, suggest that selective social mobility cannot account for inequalities in mortality by social class. ${ }^{37-39}$

Of the health related behaviours the role of smoking was investigated through separate analyses for unrelated causes of death, through analysis of mortality among subjects who had never smoked, and through controlling for smoking in proportional hazards models. While smoking is clearly an important cause of mortality, and the higher prevalence of smoking among men without cars and the lower grades will contribute to their increased mortality, it is not a sufficient explanation. Plasma cholesterol concentrations were unrelated to car ownership and were higher among the higher grades. Other dietary factors and health related behaviours could not be examined.

In the British Regional Heart Study much of the social class differentials in ischaemic heart disease incidence were accounted for by smoking and blood pressure differences (the manual groups had lower mean cholesterol levels than the non-manual groups). ${ }^{40}$ It was suggested that the inaccuracy inherent in using single measurements of risk factors as proxy measures of lifetime exposure may produce the residual associations between social class and ischaemic heart disease incidence after adjustment for risk factors. The use of imprecise measures of exposure will of course prevent full adjustments to be made, but it is also the case that the use of crude markers of socioeconomic position may mask considerably stronger underlying relationships between socioeconomic position and ischaemic heart disease incidence. The imprecision in both measurements of risk factors and in the indexing of socioeconomic position must be taken into account if this question is to be explored further.

We can say little about specific aspects of the material and social conditions of life for which employment grade and car ownership are proxy measures. It has been postulated that deprivation around infancy and childhood increases later risk of mortality from cardiovascular disease ${ }^{19} \mathrm{Height}$ in adulthood reflects conditions in early life and both higher grade and car ownership are associated with greater height. Men without cars and subjects in lower employment grades may therefore have experienced more deprivation in early life. Height was unrelated to mortality from neoplasms, a finding in line with those of a study which related birth weight and growth in the first year of life to mortality in adulthood. ${ }^{22}$ The differentials in mortality for neoplasms, which do not appear to be related to early life deprivation, were similar to those for cardiovascular disease and other causes. Furthermore controlling for height had little effect upon the mortality differentials by grade and car ownership status from all causes and from the major cause groups. On the other hand the relationship between height and mortality is partially accounted for by socioeconomic position in adulthood. Thus material conditions in childhood, to the extent to which they are indexed by adult height, seem to explain little of the mortality differentials related to socioeconomic position. Conversely, the association between height and mortality is partially due to the relationship between height and socioeconomic position in adult life. In the present data set, socioeconomic position in adult life was categorised into groups which were powerful discriminators of mortality risk. In studies which use less precise indices, relationships between markers of early life experience and mortality may suffer from residual confounding by adult socioeconomic position.

The potential explanations for the mortality differentials cannot be evaluated fully in the present data. However the similarity of the differentials across the major cause groups renders a single explanation unlikely. Low social class similarly increases risk from most causes of death. ${ }^{1041}$ When attempting to account for the association between socioeconomic position and death rates from any particular cause this general relationship must also be considered.

Men in the Whitehall study were mainly from non-manual social class groups. In $1968,49 \%$ of the population of Britain had access to a car, ${ }^{42}$ whereas $72 \%$ of the subjects in this study were car owners. The study sample were therefore a relatively privileged group-in $1972,18 \%$ of unskilled and $31 \%$ of semiskilled manual workers had cars, ${ }^{43}$ compared to $34 \%$ of the other grades civil servants. It is probable that the range of material well being within this sample does not match that seen in the general population, yet the mortality gradients are considerably greater than those seen in analysis by social class. This could reflect well stratified recruitment of higher mortality cohorts into lower civil service grades, and within these grades into lower income jobs, leading to less car ownership. This may be part of the explanation of the current findings, but it is also likely that grade and car ownership produce better classification of the socioeconomic position of subjects than does the Registrar General's social class system, which in turn leads to greater mortality differentials. A host of factors in the social environment may influence health but are only poorly indexed by social class-earnings, wealth possession, social status, education, position in the labour market, chance of unemployment, housing quality, hours and conditions of work, and expenditure on housing, heating and clothing. ${ }^{44}$ The roughness of the Registrar General's social class categorisation was recognised at the time of its inception, ${ }^{45}$ and other studies have shown that refinements produce larger differentials. The use of social class in conjunction with other indices of socioeconomic position is advisable, for if inequalities in health are underestimated, the degree to which public health policy takes account of them will be inadequate. 
1 Stevenson THC. The social distribution of mortality from different causes in England and Wales, 1910-12. Biometrika 1923; 15: 382-400.

2 Stevenson THC. The vital statistics of wealth and poverty. $f$ $R$ Stat Soc 1928; 91: 207-20.

3 Registrar General. Supplement to the 75th Annual Report of the Registrar General for England and Wales, Part IV. Mortality of men in certain occupations in the three years Mortality of men in certain occupations in the
1910,1911 and 1912 . London: HMSO, 1923.

$4 \mathrm{Logan}$ WPD. Social class variations in mortality. $\mathrm{Br} \mathcal{F}$ Prev Soc Med 1954; 8: 128-37.

5 DHSS. Inequalities in health. London: HMSO, 1980.

6 Leete R, Fox AJ. Registrar-General's social classes: origins and uses. Population Trends 1977; 8: 1-7.

7 Jones IG, Cameron D. Social class analysis: an embarrassment to epidemiology. Community Med 1984; 6: 37-46.

8 Alderson M. A comment on social class analysis. Community Med 1984; 6: 1-3.

9 Fox AJ, Jones DR. Authors' reply. $\mathcal{f}$ Epidemiol Community Health 1985; 39: 275-6.

10 OPCS. Occupational mortality, decennial supplement 1970 72. DS no. 1, London: HMSO, 1978.

11 Morris JN. Social inequalities undiminished. Lancet 1979; i: $87-90$.

12 Fox AJ, Goldblatt P. Socioeconomic differentials in mortality 1971-75. (Office of Population Censuses and Surveys Series LS1). London: HMSO, 1982.

13 Leon D. Social distribution of cancer 1971-75. (Office of Population Censuses and Surveys Series LS3). London: HMSO, 1988.

14 Moser KA, Pugh HS, Goldblatt PO. Inequalities in women's health: looking at mortality differentials using an alternative approach. $\mathrm{Br}$ Med $\mathcal{f} 1988 ; 296: 1221-4$.

15 Marmot MG, Rose G, Shipley M, Hamilton PJS. Employment grade and coronary heart disease in British civil servants. F Epidemiol Community Health 1978; 32: 244-9.

16 Rose G, Marmot MG, Social class and coronary heart disease. Br Heart $\mathcal{7} 1981$; 45: 13-19.

17 Marmot MG, Shipley MJ, Rose G. Inequalities in deathspecific explanations of a general pattern? Lancet 1984; i: $1003-6$.

18 Goldblatt PO. Mortality differences at working ages: the use of generalised linear models to compare measures. (Social Statistics Research Unit working paper No. 53.) London: City University, 1987.

19 Forsdahl A. Are poor living conditions in childhood and adolescence an important factor for arteriosclerotic heart adolescence an important factor for arteriosce

20 Rona RJ. Genetic and environmental factors in the control of growth in childhood. Br Med Bull 1981; 37: 265-72.

21 Kuh D, Wadsworth M. Parental height: childhood environment and subsequent adult height in a National Birth Cohort. Int $\mathcal{Y}$ Epidemiol 1989; 18: 663-8.

22 Barker DJP, Winter PD, Osmond C, Margetts B, Simmonds SJ. Weight in infancy and death from ischaemic heart disease. Lancet 1989; ii: 577-80.
23 Nystrom Peck AM, Vagero DH. Adult body height, selfperceived health and mortality in the Swedish population. $\mathcal{F}$ Epidemiol Community Health 1989; 43: 380-4

24 Reid DD, Brett GZ, Hamilton PJS, Jarrett RJ, Keen H, Rose G. Cardiorespiratory disease and diabetes among middle-aged male civil servants. Lancet 1974; i: 469-73.

25 Rose GA, Blackburn H. Cardiovascular survey methods. Geneva: World Health Organization, 1968.

26 Doll R, Peto R. Mortality in relation to smoking: 20 years' observations on male doctors. $\mathrm{Br}$ Med F 1976; ii: 1523-36.

27 Shinton R, Beevers G. Meta-analysis of relation between cigarette smoking and stroke. Br Med $\mathcal{F}$ 1989; 298: 789-94.

28 Hammond EC. Smoking in relation to the death rates of one million men and women. In: Haenzel W, ed. Epidemiological approaches to the study of cancer and other chronic diseases (National Cancer Institute Monograph No. 19). Bethesda, MD: National Cancer Institute, 1966: 127-204.

29 IARC. Monographs on the evaluation of the carcinogenic risk of chemicals to humans, vol 38. Tobacco smoking. Lyon: IARC, 1986.

30 Cox DR. Regression models and life-tables. $\mathcal{F}$ Stat Soc Ser $B$ 1972; 34: 187-220.

31 Townsend $\mathrm{P}$, Phillimore $\mathrm{P}$, Beattie A. Health and deprivation: inequality and the north. London: Croom Helm, 1988.

32 Carstairs V, Morris R. Deprivation: explaining differences in mortality between Scotland and England and Wales. $\mathrm{Br}$ Med f 1989; 299: 886-9.

33 Carstairs V, Morris R. Deprivation and mortality: an Carstairs V, Morris R. Deprivation and mortality: an

34 Blane D. An assessment of the Black Report's explanations of health inequalities. Sociology Health Illness 1985; 7: of health

35 Stern J. Social mobility and the interpretation of social class mortality differentials. F Soc Policy 1983; 12: 27-49.

36 Illsley R. Occupational class, selection and the production of inequalities in health. $Q \mathcal{f}$ Soc Affairs 1986; 2: 151-65.

37 Goldblatt $P$. Changes in social class between 1971 and 1981 could these affect mortality differential among men of working age? Population Trends 1988; 51: 9-17.

38 Goldblatt P. Mortality by social class, 1971-85. Population Trends 1989; 56: 6-15.

39 Wilkinson RG. Occupational class, selection and inequalities in health: a reply to Raymond Illsley. $Q \mathcal{F} S o c$ Affairs 1986; 2: 415-22.

40 Pocock SJ, Shaper AG, Cook DG, Phillips AN, Walker M. Social class differences in ischaemic heart disease in British men. Lancet 1987; ii: 197-201.

41 Office of Population Censuses and Surveys. Occupational mortality decennial supplement 1979-80; 1982-3. London, mortality decen

42 Central Statistical Office. Social Trends No. 2, 1971. London: HMSO, 1971.

43 OPCS. General Household Survey for 1972. London: HMSO, 1974.

44 Reid I. Social class differences in Britain: life-chances and life-styles. Glasgow: Fontana Press, 1989.

45 Stocks P. Discussion on Dr Stevenson's Paper. F R Stat Soc 1928; 91: 225-6. 